\title{
Development of new medical treatment for epithelial ovarian cancer recurrence
}

\author{
Rosanna Mancari ${ }^{1}$, Giuseppe Cutillo ${ }^{1}$, Valentina Bruno ${ }^{1}$, Cristina Vincenzoni ${ }^{1}$, Emanuela Mancini ${ }^{1}$, \\ Ermelinda Baiocco ${ }^{1}$, Simone Bruni ${ }^{2}$, Giuseppe Vocaturo ${ }^{1}$, Benito Chiofalo ${ }^{1}$, Enrico Vizza ${ }^{1}$ \\ ${ }^{1}$ Department of Experimental Clinical Oncology, Gynecologic Oncology Unit, IRCCS Regina Elena National Cancer Institute, Rome, Italy; \\ ${ }^{2}$ Division of Obstetrics and Gynecology, Department of Molecular and Developmental Medicine, University of Siena, Siena, Italy \\ Contributions: (I) Conception and design: R Mancari, E Vizza; (II) Administrative support: R Mancari; (III) Provision of study materials or patients: \\ R Mancari; (IV) Collection and assembly of data: R Mancari; (V) Data analysis and interpretation: E Vizza, G Vocaturo; (VI) Manuscript writing: All \\ authors; (VII) Final approval of manuscript: All authors. \\ Correspondence to: Rosanna Mancari. Department of Experimental Clinical Oncology, Gynecologic Oncology Unit, IRCCS Regina Elena National \\ Cancer Institute, Rome, Italy. Email: rosanna.mancari@ifo.gov.it.
}

\begin{abstract}
Epithelial ovarian cancer (EOC) is the scariest gynaecological cancer. Many advances have been done with evolving knowledge, leading to the introduction of new drugs, most in maintenance setting. The antiangiogenic Bevacizumab and the three approved PARP-inhibitors-olaparib, niraparib and rucaparibare gradually improving PFS of patients with EOC, with initial effects on OS too. But recurrence is still a heavy sentence and lethality continues to be high. Ovarian cancer is a complex disease, with different clinical presentation, histological aspect, and molecular expression, leading to disappointing results, when using a single drug. Implementation of biobanking and analysis of patients' tumour samples, before starting a treatment, could be a promising way to better understand molecular aspects of this disease, to identify markers predictive of response and to allow a better use of experimental drugs, as immunomodulators, targeted therapies, and combinations of these, to fight tumour growth and clinical progression. We reviewed the literature on the updated treatments for recurrent ovarian cancer, summarizing all the available drugs and combinations to treat patients with this diagnosis, and focusing the attention on the new approved molecules and the contemporary Clinical Trials, investigating new target therapies and new associations.
\end{abstract}

Keywords: Ovarian cancer; chemotherapy; recurrence; PARP inhibitors; BRCA mutation

Submitted Mar 27, 2020. Accepted for publication Jul 01, 2020.

doi: 10.21037 /gs-20-413

View this article at: http://dx.doi.org/10.21037/gs-20-413

\section{Introduction}

Ovarian cancer is the most lethal gynaecologic neoplasia, and the fifth cause of cancer death among women (1). Most patients are diagnosed with stage III and IV disease, with a bad prognosis. Around $90 \%$ of cases are epithelial ovarian cancer (EOC): in this review we will use this term to include also Fallopian Tube and Primary Peritoneal Cancer. Highgrade serous carcinomas (HGSC) is the most common histotype (75\%) (2); its response rate to the first platinumbased chemotherapy is high, even if more than two third of patient usually relapse $(3,4)$. Low-grade serous carcinomas (LGSC) tend to behave with an indolent course and to have a lower response-rate to platinum agents; a high proportion of LGSCs have estrogen receptor (ER) and progesterone receptor (PR) expression, and hormonal therapy, like Anastrozole, seems to provide clinical benefit in $>70 \%$ of relapses (5).

During the last decades an improvement in treatment quality resulted in a decrease in cancer mortality, likely due to improvement of the treatment of recurrent disease (6).

The objective of the study is to analyse the landscape of actual treatment of recurrent ovarian cancer, summarizing the achieved knowledge in standard treatment and focusing the attention on recent findings improving medical 
Table 1 Factors that guide physician in the choice of medical treatment for patient with ROC

Tumour-related factors
Platinum-free interval
Histotype
Molecular characteristics of the disease
BRCA mutation or HRD
Patient-related factors
Number of previous lines of chemotherapy
Type of previous chemotherapy
Eventual residual toxicities
Nutritional status
Symptoms related to the presence of disease
Patient's desire

treatment and on new perspectives in this setting.

The management of patients with recurrent EOC is complex and has to take into account many different factors (Table 1). In the recent past decision-making process was based mainly on the so-called "platinum-sensitivity", depending on the period lasting from the end of first-line therapy to the subsequent relapse, with an arbitrary cutoff of 6 months to differ platinum-resistant from platinumsensitive patients $(7,8)$.

The recent acquisitions led physicians to accept the idea that response to platinum is a continuous variable and that patients with recurrent disease have different prognosis, depending also from histology, site of recurrence and number of localizations-conditioning the possibility to offer secondary cytoreduction or not-previous therapies, patient's conditions due to comorbidities or to symptomatic relapse, patient's desire, residual side-effects from previous treatments, genetic factors and so on (9).

'Platinum sensitivity and platinum resistance' have recently acquired a probabilistic meaning, based on a likely good or poor response to platinum therapy, respectively.

Anyway platinum-free interval (PFI) depicts a useful clinical parameter, frequently used in clinical trials, since patients with platinum-sensitive relapse has a better prognosis, due to a statistically better response to platinum agents than the platinum-resistant patients (10).

\section{Medical treatment for platinum-resistant and platinum-refractory relapse}

Around $15-20 \%$ of patients experience disease progression a short period after completing a platinum-based chemotherapy, or fail to respond at all to this treatment. No validated biomarkers is known to predict likelihood of primary platinum-resistant or platinum-refractory disease. Only histotype gives a similar indication: lowgrade serous and mucinous ovarian cancer, as well as clear cell and endometrioid ovarian carcinomas are less chemoresponsive, with a different prognosis, although they are often diagnosed at an early stage.

Platinum resistance may be distinguished into primary platinum resistance, that is an intrinsic condition, proper of the tumour, and occurs during first-line therapy-leading to progressive disease and worsening conditions during therapy or shortly afterwards-and secondary platinum resistance, that is an acquired condition, emerging after a previous response to platinum therapy.

Occasionally PFI $<6$ months is not predictive of absence of response to following platinum-based therapy (11), especially when patient experiments an intercurrent response to other drugs, and she may be candidate for further platinum followed by maintenance therapy. Particularly from BRCAmutated patients, we may be expected to have an additional response to platinum rechallenge therapy (12), and PARP inhibitors seems to be active both in platinum resistant and in platinum sensitive patients (13).

When patient is not eligible to platinum rechallenge for early relapse, with symptomatic relapse or worsened performance status, the objective of treatment should change and be focused on quality of life (QoL) and control of symptoms, trying to limit side effects: monotherapy with non-platinum agents should be preferred, in sequential way, with weekly paclitaxel, PLD, gemcitabine, topotecan or etoposide $(14,15)$; no RCT (randomized Clinical Trial) is available to compare them (16). The addition of bevacizumab to non-platinum, may improve PFSwith a median PFS of 6.7 months versus 3.4 months with chemotherapy alone-and contribute to reduce ascites, gastro-intestinal symptoms and pleural effusion $(17,18)$. The US Food and Drug Administration and the European Commission approved bevacizumab combined with chemotherapy for platinum resistant ROC on the basis of results from AURELIA Trial. 
Table 2 Approved PARP-inhibitors for HGSOC in clinical practice

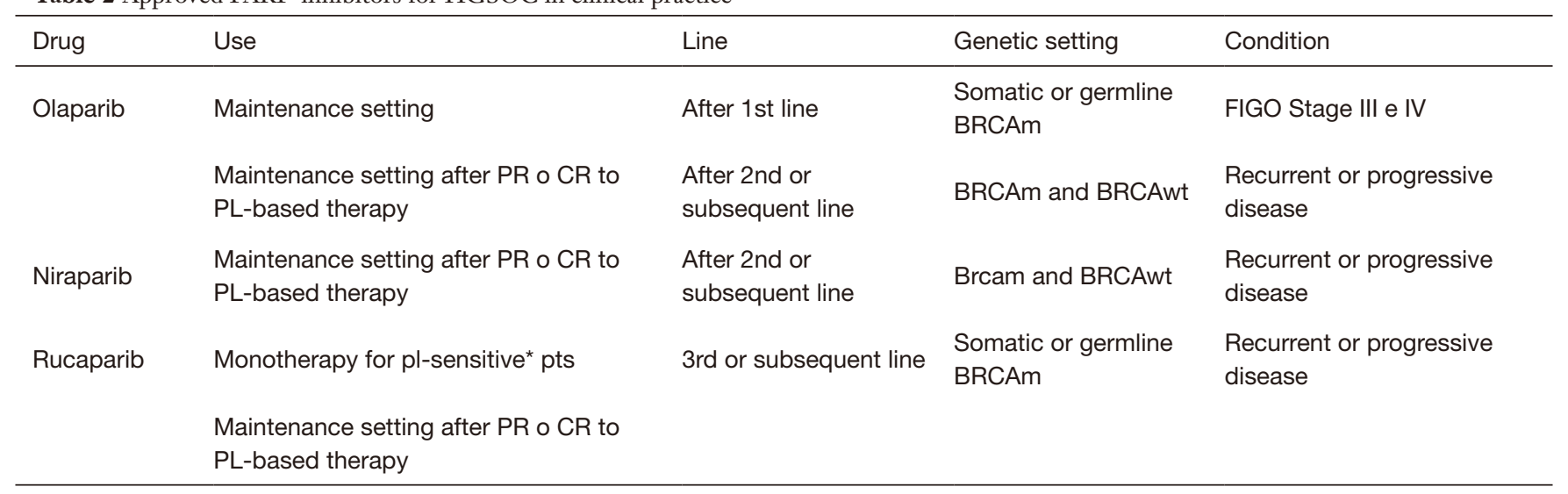

*, if patient cannot use platinum. PR, partial response; CR, complete response; PL-based, platinum-based; Pts, patients.

\section{Medical treatment for platinum-sensitive relapse}

Every physician may experience a difference in response rate of patients with recurrence of platinum-sensitive disease, usually proportional to the length of PFI. Depending on patient's conditions, single-agent carboplatin or association with other drugs may be proposed.

The first phase III RCT dealing with this choice was ICON4/OVAR 2.2, that compared the efficacy and tolerability of platinum alone to platinum and paclitaxel therapy (3). OS and PFS were improved in the second group, with acceptable toxicity, and the association became the standard of treatment in first platinum-sensitive relapse, if general conditions were valid, no residual peripheral neuropathy was present and alopecia was accepted.

In the AGO-OVAR-2.5 trial gemcitabine and carboplatin were compared with carboplatin monotherapy, and an increase of PFS was documented with the combination, that has been well tolerated, even without increase in OS (19).

The third experimented combination was carboplatin and pegylated liposomal doxorubicin (PLD), compared with carboplatin-paclitaxel: the new combination showed lower toxicity with a median PFS slightly superior (CALYPSO Trial) (20). Even if the carboplatin-gemcitabine association and the carboplatin-PLD association didn't demonstrate any difference in OS in comparison with carboplatinPaclitaxel regimen, their lower toxicity could suggest them as preferable regimens in this population.

In patients with PFI between 6 and 12 months OVA-301 trial compared PLD alone with PLD and Trabectedin (21): the combination showed a benefit in terms of PFS and OS, and an additional as well as interesting outcome, emerging from this trial, was the possibility of delaying the following line of platinum therapy, assuming that this artificial prolongation of platinum interval could increment the response to this drug (22). In a short period, we should receive the results of Inovatyon study (NCT01379989), planned to compare carboplatin-PLD with PLDTrabectedin, to understand if the best option for patient with 6-12 months relapse is to use platinum or nonplatinum drugs.

More advances were obtained with OCEANS Study, a phase III RCT, that demonstrated the superiority of the association of Carboplatin, Gemcitabine and Bevacizumab in terms of PFS (12.4 vs. 8.4 months, $\mathrm{P}<0.001)$ on the association of Carboplatin and Gemcitabine, without difference in OS $(23,24)$. The lack of difference in OS could have been influenced from the short-lasting therapy with Bevacizumab and from the frequent use of Bevacizumab in subsequent line of therapies. The results of this study allowed the registration of bevacizumab in combination with gemcitabine and carboplatin, to treat patients with first recurrence of platinum-sensitive ovarian cancer.

Bevacizumab is a monoclonal antibody directed against VEGF and its action, disrupting angiogenesis-that has a key-role in survival, replication and metastasis of cancer cells-has demonstrated to have a relevant clinical impact in many subgroups of patients, both with platinum-sensitive and with platinum-resistant ovarian cancer patients. Toxicity profile of Bevacizumab is now well known, with hypertension and proteinuria, that are frequent but easily manageable.

Another study testing efficacy of Bevacizumab was MITO-16, a phase III RCT (NCT01706120) that evaluated the retreatment with Bevacizumab and chemotherapy in 
patients who already received bevacizumab in first-line therapy and experimented a platinum-sensitive recurrence: the results are encouraging, for the improvement in PFS (11.8 vs. 8.8 months, $\mathrm{P}<0.0001$ ), while data for OS is not available yet (25).

\section{Recent findings improving medical treatment: PARP inhibitors}

PARP inhibitors (PARPi) are one of the most encouraging finding of clinical research in EOC in the last decades, and they are going to change natural history of this disease. They include Olaparib, Niraparib, Rucaparib, Talazoparib and Veliparib. The first three have been widely analysed (Table 2), showing their relevant anticancer activity in BRCA mutant (BRCAm) patients; the different trials, some of that still ongoing, demonstrated later their growing interest also in BRCA wild type (BRCAwt) patients with platinum sensitivity (26).

Nearly $20 \%$ of all EOC present a germline BRCA mutation, $6 \%$ a somatic BRCA mutation and approximately $50 \%$ of all HGSCs seems to have HRD (Homologous Recombination Deficiency) $(27,28)$. BRCAness phenotype in HGSOC patients correlates with response to platinumderived drugs and with PARP inhibitor.

PARPi express their potent antitumor activity killing ovarian cancer cells with BRCA1 or 2 deficiency (29-33). Nevertheless, PARPi are active, even if with a lower strength, also in BRCAwt HGSOC, mainly in 'platinumsensitive' recurrence (12).

PARP enzymes are necessary for cell survival, since they are involved in DNA repair; PARPi express its lethality inducing DNA damage, that is usually repaired by Homologous Recombination (HR) system and that is defective in BRCAm or HRD cells, with consequent cellular apoptosis $(26,34,35)$.

While Study 19 and SOLO2 studied olaparib and led the approval of this drug only for BRCAm patients, NOVA and ARIEL3, studying Niraparib and Rucaparib respectively, extended their indication even to BRCAwt patients as long as it was documented platinum-sensitivity, and both used BRCA status and HRD/LOH (loss of heterozygosity) status as predictor of response to treatment.

\section{Olaparib}

Olaparib is a well-known oral PARP-inhibitor that received FDA accelerated approval and EMA approval in 2014 for germline BRCAm HGSOC patients with platinum-sensitive recurrence $(30,31,36-40)$.

The milestone study that led to Olaparib FDA registration was 'Study 19', a randomized, placebo controlled, double-blind, phase 2 study evaluating maintenance treatment with olaparib versus placebo after partial or complete response to chemotherapy (41). PFS was primary endpoint and it resulted significantly longer in the treatment group (8.4 vs. 4.8 months; HR 0.35; $\mathrm{P}<0.001$ ); time to first subsequent treatment as time to subsequent relapse were also improved. These promising results were only a part of the real effect, since the study was originally designed to include both patients with and without BRCA mutations. A subsequent analysis was performed to evaluate the benefit of the experimental drug on BRCA-mutated patients; this subgroup of patients had further benefits in all end points: median PFS of 11.2 vs. 4.3 months, with impressive HR $=0.18(\mathrm{P}<0.0001)$. BRCAwt patients also benefited from olaparib maintenance, although the benefit was much lower (7.4 vs. 5.5 months).

A confirmatory phase III study, Solo2, enrolled only patients with known or suspected BRCA-mutation and had at least PR (partial response) following completion of platinum-based chemotherapy. SOLO2 confirmed that PFS in the olaparib group was significantly longer than placebo (19.1 vs. 5.5 months, HR 0.30, 95\% CI, 0.22-0.41; $\mathrm{P}=0.0001)$ (36).

Olaparib is generally well tolerated: the most common adverse events are nausea, fatigue, vomiting and anemia. One patient in Solo2 Study developed acute leukemia (AML) with a fatal outcome. No significant worsening of QoL has been described in both studies (37) and the 'Time without symptoms of disease or toxicity' (TWIST) in Solo2 has been longer in Olaparib group than in placebo group (13.5 vs. 7.1 months). Solo1 study results finally allowed the approval of Olaparib for maintenance therapy in patients with newly diagnosed advanced OC, after first line of chemotherapy, since olaparib provided a substantial benefit with regard to PFS among women with BRCA1/2 mutation (38).

\section{Niraparib}

Niraparib is a particularly potent inhibitor of PARP-2. In 2017 FDA approved it for maintenance therapy in all women with recurrent EOC, after response to platinumbased chemotherapy.

NOVA trial was the pivotal phase III RCT, that showed a prolongation of PFS with Niraparib versus placebo in all 
the three studied populations: gBRCAm patients (PFS of 21.0 versus 5.5 months, $\mathrm{HR}=0.27, \mathrm{P}<0.0001$ ), non-BRCAm with HRD tumors (PFS of 12.9 versus 3.8 months, HR $=0.38, \mathrm{P}<0.0001)$, and non-gBRCAm cohort $(\mathrm{PFS}$ of 9.3 versus 3.9 months, $\mathrm{HR}=0.45, \mathrm{P}<0.001$ ) (42).

NOVA has been able to demonstrate the benefit of a PARPi in recurrent platinum-sensitive EOC beyond BRCA mutation and these results have been confirmed also in heavily pretreated EOC patients (43). QoL seems not to be altered from Niraparib use (44).

Results regarding OS are still immature and have not yet been reported until now; at interim analysis Niraparib showed a trend towards prolonging OS versus placebo. Toxicity profile of Niraparib is similar to the other PARPi, however there are some that are peculiar of this drug, as Grade 3/4 thrombocytopenia, occurred in one third of cases, more frequent during the first cycles, and Grade 3/4 hypertension, in $9 \%$ of patients (45). Thrombocytopenia has been studied and can be reduced with an accurate choice of initial dosage, depending on patient's body weight and basal platelet count (46); hypertension may be easily prevented with a care monitoring of blood pressure, to start an appropriate therapy as needed.

Unlike the other PARPi, niraparib has no effect on cytochrome $\mathrm{P} 450$ enzymes, and the probability of interaction with concomitant drugs is less probable; another unique characteristic is the ability to cross the blood brain barrier, with a possible therapeutic effect on this organ, mostly after radiotherapy (45).

\section{Rucaparib}

PARP-inhibitor Rucaparib received FDA approval in 2016 as monotherapy, and in 2018 as maintenance therapy (47). The first breakthrough indication is valid after two previous lines of therapy, for patients with platinum-sensitive EOC relapse, and documented BRCA mutation, based on the results of the ARIEL-2 phase II study (48). The confirmatory phase III study was named ARIEL-3 (33).

ARIEL2 was designed to identify molecular predictors of response to treatment with Rucaparib, in patients with platinum-sensitive recurrence of disease, testing a new HRD assay; in ARIEL2 Part 1 patients were stratified into one of three HRD subgroups: BRCAm (germline or somatic), BRCAwt and LOH high, or BRCAwt and $\mathrm{LOH}$ low. Primary objective was PFS, that resulted 16.6 months in first group, 13.6 months in the second and 10.8 months in the third group. This result support the important role of high genomic $\mathrm{LOH}$ as a predictive biomarker for sensitivity to treatment with Rucaparib (48).

Furthermore, ARIEL2 Part 1 showed that the mutation of RAD51C/D, another recombination-related gene, may be correlated with high genomic LOH in BRCA wild-type tumors, hence with rucaparib response.

ARIEL2 Part 2 demonstrated the major benefit in BRCAm patients, with platinum-sensitive disease, compared to the platinum-resistant/refractory patients $(49,50)$. To confirm the predictive value of the HRD assay tested in ARIEL2, it was applied in ARIEL3, designed as doubleblind, placebo-controlled phase III RCT, comparing Rucaparib maintenance versus placebo, after partial or complete response to last platinum-based therapy.

The three cohorts of patients treated with Rucaparib had better response than placebo, in favour of BRCAm group, with PFS of 16.6 vs. 5.4 months; among patients with a HRD disease, median PFS was 13.6 versus 5.4 months. For the Intention To Treat (ITT) population, the median treatment duration was 10.8 months for Rucaparib group vs. 5.4 months $(\mathrm{P}<0.0001)$.

The most common grade 3 adverse events have been anemia and elevation in alanine aminotransferase or aspartate aminotransferase, both easy to monitor and to manage, by adjusting the dosage of Rucaparib.

Considerations about the choice of which PARPi to prescribe should be based on the side effect profile of the drug, that may exacerbate any pre-existing toxicities or comorbidities, physician experience with various PARPi, and patient's BRCA status.

\section{Other PARPi: veliparib and talazoparib}

Veliparib and talazoparib are other potent promising PARPi: RCT in ovarian cancer are ongoing with both drugs $(51,52)$.

Veliparib, a more recent PARPi, has demonstrated a response rate (RR) of $26 \%$, as monotherapy in relapsed BRCAm EOC, with a median PFS of 18.8 months in a phase 2 study (53). Veliparib has not been approved yet, but there are currently promising results available in preclinical and early clinical settings. Moreover in an international, phase III, placebo-controlled RCT, the efficacy of veliparib has been studied in first-line therapy for stage III or IV HGSOC, and continued as maintenance monotherapy (VELIA/GOG-3005, NCT02470585). The association therapy led to significantly longer PFS than chemotherapy alone: in the BRCAm cohort median PFS was 34.7 vs. 
22 months; in the HRD cohort, it was 31.9 vs. 20.5 months; in ITT population, it was 23.5 vs. 17.3 months (hazard ratio, 0.68; $\mathrm{P}<0.001)(54)$.

Talazoparib has been studied in phase I trials and showed some clinical activity in relapsed BRCAm EOC. Actually more combination are under investigation, in association with Avelumab (NCT03330405) and with Pembrolizumab and ZN-c3 (NCT04158336).

\section{New perspectives in ROC}

\section{Immunotherapy}

Immunotherapy is emerging as a promising approach to many tumours; in ovarian cancer treatment it is actually under investigation and limited only to clinical trials.

EOC, mostly if BRCA mutation is present, is characterized by a higher mutational load, and cancer cells expose neo-antigens, that stimulate the recruitment of TILs (tumor-infiltrating lymphocytes), mainly CD3+ and CD8+ and elevated expression of programmed death 1 (PD-1) and programmed death-ligand 1 (PD-L1), representing an ideal subset for treatment with immune checkpoint in combination with platinum-based chemotherapy or PARP inhibitors (55). Several trials have the objective to investigate the role of immune checkpoint inhibitors, some of them in combination with PARP inhibitors, both in first line and in recurrence setting (56-59).

Most of them are based on anti PD-1/PD-L1 pathway inhibitors and they can be used in combination with standard therapy. Some data have shown that the PD-1/ PD-L1 pathway blockade may be more effective in specific histotype, as the endometrioid one.

\section{Nivolumab}

Nivolumab is a monoclonal antibody that blocks PD-1 signaling targeting PD-1 receptor. In 2015 patients with recurrent EOC were treated with two different dosage in a phase II trial, with nivolumab 1 and $3 \mathrm{mg} / \mathrm{kg}$ : best overall response was $15 \%$ (primary end point) and two patients in $3 \mathrm{mg} / \mathrm{kg}$ cohort experienced a complete and durable response (56).

In a phase I study the combined treatment with nivolumab and cisplatin inhibited platinum-resistant OC cancer cells, inducing cell apoptosis and inhibition of ADAM17 expression $(56,60)$.

More trials with nivolumab are ongoing: two of them are waiting for results: one (NCT02737787) where nivolumab has been tested with investigational WT1 vaccine, and a randomized phase II trial (NCT02498600) to analyse the role of nivolumab with or without ipilimumab.

\section{Pembrolizumab}

Pembrolizumab is another anti-PD-1 antibody, evaluated in a phase Ib study (KETNOTE 028) (57), with initial evidence of activity, followed by a phase II study with Pembrolizumab monotherapy in subjects with advanced ROC showed modest activity, with ORR of $4.1 \%$ and PFS of 2.1 months, where higher PD-L1 expression was correlated with better response (KEYNOTE-100). A phase III study is now enrolling BRCAwt patients in a RCT for advanced OC in first line therapy with standard chemotherapy, with or without Pembrolizumab, followed by maintenance with olaparib or placebo (KEYLYNK-001/ ENGOT-ov43) (NCT03740165).

\section{Avelumab}

Another monoclonal anti-PD-L1 antibody is Avelumab: preliminary results of a phase IB study (NCT01772004) showed activity of avelumab in patients with refractory ROC, with a ORR of $12.3 \%$ in PD-L1-positive tumours (58). A phase III trials of avelumab for frontline therapy in combination with carboplatin and paclitaxel (Javelin ovarian 100) has been discontinued for the disappointing results. In another Phase 3 clinical trial, dedicated to recurrent platinum-resistant/refractory disease (Javelin ovarian 200), Avelumab did not induce a significant improvement in OS or PFS versus PLD alone (NCT02580058).

\section{Ipilimumab}

Ipilimumab, tested in different diseases, is a monoclonal antibody that blocks the immunosuppressive signal by cytotoxic T-lymphocyte antigen (CTLA-4). Its efficacy in recurrent platinum-sensitive $\mathrm{ROC}$ was evaluated in a phase II study (NCT01611558) with an ORR of $10.3 \%$.

\section{New antiangiogenic drugs: antiangiogenesis beyond bevacizumab}

Angiogenesis, that is a fundamental step for tumour grow, spread and metastatization, is dependent from different key factors: vascular endothelial growth factor (VEGF), plateletderived growth factor (PDGF), angiopoietin-Tie2 receptor, and fibroblast growth factor (FGF) (61). To antagonize angiogenesis is necessary to target these key factors. 


\section{Cediranib}

Cediranib is an oral tyrosine kinase inhibitor of VEGF receptor and c-KIT and has shown antitumor activity in recurrent EOC in many studies. ICON6 was a phase III RCT for platinum-sensitive relapsed ovarian cancer, where PFS has been significantly improved in arm A (chemotherapy/placebo, followed by placebo versus arm $\mathrm{C}$ (chemotherapy/cediranib followed by cediranib) with a value of $11.0 v s .8 .7$ months $(\mathrm{P}<0.0001)$ (62). OS moved from 27.3 vs. 19.9 months, but this improvement was not statistically significant $(\mathrm{P}=0.21)$ (63). Diarrhea, neutropenia, hypertension, voice changes and hypothyroidism were the most common side effects, that were responsible of $40 \%$ of discontinuation in Arm C (62); nevertheless, QoL seems not to be negatively influenced from the drug (64).

\section{Inhibitor of angiopoietin: trebananib}

Trebananib inhibits angiopoietin-1 and -2 , which are involved in the mechanism of angiogenesis, and they exert a crucial roles in tumour progression (65).

TRINOVA-1 was a double-blind, placebo-controlled phase 3 RCT, evaluating patients with recurrent disease $<12$ months (66). Patients were randomized to receive weekly paclitaxel, together with trebananib or placebo. PFS was longer in the trebananib group (7.2 vs. 5.4 months, $\mathrm{P}<0.001$ ), while OS was not different between the two groups (67).

Unfortunately, TRINOVA-2 and Trinova-3 trials, in ROC or in first line of treatment, respectively, had disappointing results (68), since neither OS or PFS have been improved.

Given the mechanism of action, that differs from antiVEGF receptors, the adverse events of trebananib are distinct and safety profile has been not favourable: the main side grade 3 effects were diffuse oedema (more than $60 \%$ ), ascites (30\%) and vomiting (45\%) (68).

\section{Pazopanib}

Pazopanib is an oral multitarget kinase inhibitor of VEGFR, PDGFR and c-kit, who showed promising activity as monotherapy in a phase II study conducted in patients with ROC (69). In a following phase II RCT the combination of pazopanib plus paclitaxel has not demonstrated superior efficacy to paclitaxel alone in women with ROC.

After the initial encouraging of MITO11 Study for patients with platinum-resistant/refractory ROC (70), a phase 3 study (AGO-OVAR16) of maintenance pazopanib versus placebo was planned for patients after first-line chemotherapy for advanced ovarian cancer. Although pazopanib prolonged PFS, this was not associated with improvement in median OS, and grade 3-4 adverse events were more common in the treatment group than in the control one, with neutropenia, fatigue, leucopenia, hypertension, raised AST and ALT and anemia (71). For both reasons Pazopanib did not find any clinical application for ROC.

\section{Combinations}

Based on the hypothesis that the interference of PARPi on Base Excision Repair (BER) system could synergize with chemotherapy, many combination trials has been started with olaparib or veliparib and chemotherapy $(72,73)$; however full doses of both regimens are conditioned by overlapping toxicities (74-79). Alternatively different combinations were tested with PARPi and antiangiogenics or other drugs, trying to obtain a potentiated effect, with lesser toxicities (40).

First evidences of activity in OC have been demonstrated in phase I studies of olaparib in combination with the PI3K inhibitor BKM120 (NCT01623349) and the AKT inhibitor AZD5363 (NCT02208375).

\section{Combinations of immunotherapy with other drugs}

Many pre-clinical and clinical evidences support the combinations between immunotherapy, specifically PD-1inhibitors, with chemotherapy and anti-angiogenics agents such as bevacizumab. Now several trials are ongoing and we need time to wait for their results.

A single-arm phase II study of neoadjuvant chemotherapy plus durvalumab-a new antibody against PDL1—and tremelimumab, an anti-CTLA4 antibody, in the treatment of advanced-stage ovarian cancer (NCT03899610).

A similar phase I/II trial, now recruiting patient, for recurrent or refractory OC BRCAm patient, is studying olaparib with durvalumab and tremelimumab (NCT02953457).

Another ongoing phase I/II study, for advanced or recurrent $\mathrm{OC}$, with durvalumab added to olaparib or cediranib showed one PR in nine evaluable OC with the first combination and one PR in five evaluable OC patients treated with the second combination (NCT02484404).

One more RCT_-Mediola study — has the objective to compare durvalumab in combination with olaparib, and durvalumab in combination with olaparib and bevacizumab, in patient with advanced $\mathrm{OC}$ ad it is now recruiting 
(NCT02734004).

A Phase III RCT is enrolling patients with newly diagnosed advanced OC, to be treated with platinum based chemotherapy and bevacizumab with durvalumab, followed by maintenance durvalumab and bevacizumab or durvalumab, bevacizumab and olaparib (DUO-O) (NCT03737643).

IMagyn050/GOG 3015/ENGOT-OV39 is a Phase III, double blind, multicenter RCT of another anti-PD1 Atezolizumab versus placebo, administered in combination with paclitaxel, carboplatin and Bevacizumab in first line advanced OC, from which we are waiting for results.

The phase III randomized, double-blinded ATALANTE RCT has enrolled patient with platinum sensitive recurrence, receiving Atezolizumab or placebo, in combination with platinum-based chemotherapy and bevacizumab (NCT02891824).

Other combination trials include a phase II study (EORTC-1508) investigating Atezolizumab with bevacizumab or acetylsalicylic acid in platinum-resistant ROC (NCT02659384), still enrolling (80).

Further development of these combinations are needed, and they have already enriched to include other immunotherapic agents and PI $3 \mathrm{~K}$ inhibitors, based on previous studies, who showed synergistic effects (81).

\section{Combinations of PARP inhibitors and antiangiogenics}

This combination started to be explored on the basis of hypothesis that hypoxia induced by antiangiogenic agents could determine a functional weakening of HR, thus sensitising BRCAwt tumour cells to the action of PARPi. This mechanism could reveal the potential synergy of PARPi with anti-angiogenic agents (82).

A phase 2 study for patients with platinum-sensitive ROC analysed the efficacy of olaparib alone or in combination with cediranib: median PFS was significantly longer for the combination group (17.7 versus 9.0 months, $\mathrm{HR}=0.42 ; \mathrm{P}=0.005)$, without any particular advantage in BRCAm patient. The combination group demonstrated also a trend toward longer OS. Toxicities included diarrhea, hypertension, myelosuppression and fatigue (NCT01116648) (80-83).

A phase I/II AVANOVA (NCT02354131) randomized platinum-sensitive ROC patients to niraparib versus niraparib and bevacizumab: niraparib plus bevacizumab significantly improved PFS compared with niraparib alone (median PFS 11.9 vs. 5.5 months, respectively) (84). Phase II study enrolled patients with the same clinical presentation and confirmed the efficacy of this chemotherapy-free association, where the median PFS was 11.9 months with niraparib and bevacizumab versus 5.5 months with niraparib alone ( $\mathrm{HR}=0.35 ; \mathrm{P}<0,0001)$.

Based on these positive results, two phase III trials are underway in both platinum sensitive (NCT02502266) still recruiting, and in platinum resistant $\mathrm{OC}$ patient (NCT02446600), with olaparib or cediranib alone, or in combination, compared with platinum-based chemotherapy (83).

In women with platinum-sensitive ROC, ICON 9 trial, a phase III trial, is examining the role of maintenance with olaparib and cediranib versus olaparib alone, after platinumbased chemotherapy (NCT03278717).

An Italian a phase II trial randomized patient with platinum-resistant ROC to receive weekly paclitaxel versus the association of cediranib and olaparib, in continuous or intermittent schedule (Barocco Study). The group of patients treated with continuous schedule showed a promising trend towards the improvement of PFS, with the advantage of the oral administration and a good toxicity profile (85).

The phase III PAOLA-1 trial has been just closed and showed the advantage of adding olaparib maintenance to Bevacizumab following platinum-based chemotherapy in 1st line OC. The median PFS was 22.1 months with olaparib and bevacizumab versus 16.6 months with bevacizumab alone; patients with BRCAm experimented a median PFS of 37.2 months (HR $=0.33$ ) and patients with HRD without BRCAm had a median PFS of 28.1 months (HR =0.43) (86) (NCT02477644).

\section{Combination of PARPi and chemotherapy}

The association between veliparib and temozolomide versus PLD, is being tested and results are not available yet (NCT01113957).

The combination of oral cyclophosphamide and veliparib versus oral cyclophosphamide have been well tolerated and showed some clinical activity, but the addition of veliparib did not improve either the response rate or the median PFS (NCT01306032). One more ongoing trial is testing the efficacy of the association between Veliparib and carboplatin plus paclitaxel versus chemotherapy and placebo (NCT02470585).

\section{Intraperitoneal chemotherapy}

Even if scientific literature is rich in publications regarding intraperitoneal (i.p.) chemotherapy, it is not possible to 
make generalizations about this argument, since the single studies include too small groups of patients, with different schedule of treatment, not comparable each other (87-89).

HIPEC (Hyperthermic Intraperitoneal Chemotherapy) has been developed to combine the advantages of loco-regional delivery of chemotherapy with surgical radicality (90). The presumed benefit is that extensive peritonectomy allows macroscopic cytoreduction of peritoneal implants which, when combined with heated intraperitoneal chemotherapy, provides 'an avenue for further microscopic cytoreduction' (90). The biggest prospective randomized trial in the recurrent setting has been widely criticized (91-93), while a meta-analysis of retrospective studies in advanced or recurrent EOC did not show any survival advantage (94), but rather an increase in AEs (e.g., anemia, acute kidney injury), $(95,96)$ precluding HIPEC from standard-of-care treatment. For that reason, HIPEC data were not accepted within the scientific gynecologic oncology community (97).

A recent RCT had the purpose to determine the role of surgery followed by hyperthermic intra-peritoneal chemotherapy (HIPEC) versus surgery alone in patients with platinum-sensitive ROC, in terms of PFI, OS, morbidity and mortality, second recurrence pattern and QoL (HORSE NCT01539785).

Large prospective studies are required to further quantify the true efficacy of HIPEC and to compare its efficacy and compatibility with targeted therapy (e.g., bevacizumab). In the meanwhile, HIPEC should not be considered as standard therapy and be limited to well-designed prospective RCTs (98).

\section{Emerging drugs}

Novel classes of drugs, that act with different mechanisms and new targeted therapies, are under investigation, but they need time to be tested and implemented.

Antibody drug conjugates (ADC) are a novel class of antineoplastic drugs, delivering cytotoxic therapy directly within cancer cells, and seem promising in the management of OC patients.

Mirvetuximab soravtansine (IMGN853), an antibodydrug conjugate that binds to folate receptor- $\alpha$ (FR- $\alpha)$ to provide tumor-directed delivery of the potent microtubuledisrupting agent (DM4), showed promising activity in patients with ROC (99). On the basis of phase I study results, a phase III RCT of Mirvetuximab monotherapy versus standard chemotherapy has completed accrual with evidence of activity, although without reaching statistical significance (NCT02631876): a new phase II study is now starting for ROC (Mirova study), where patients with FR- $\alpha$ high will be randomly assigned to platinumbased chemotherapy versus carboplatin and mirvetuximab (NCT04274426).

Sacituzumab govitecan (SG) is a novel ADC, targeting trophoblast-antigen-2 (Trop-2), highly expressed in many tumors, to deliver SN-38, the active metabolite of irinotecan. Actually patients with solid tumour may be enrolled into SEASTAR Study, to evaluate rucaparib in combination with other anticancer agents, one of which is SG (NCT03992131).

Additional ADC are being evaluated in OC, including agents that target NaPiB2, Trop2, mesothelin, and MUC16 are in phase 1 clinical trials.

Lurbinectedin is an inhibitor of RNA polymerase II, involved in the transcription process that is over-activated in tumours; its efficacy has been investigated in various types of solid tumours and it showed activity in platinumresistant OC patients in a randomized phase II trial versus topotecan (100).

In a phase III CORAIL RCT, treated with Lurbinectedin or standard chemotherapy (investigator choice), patients did not obtain any advantage in terms of PFS, but similar efficacy and favourable safety profile has been documented (101), indicating a potential role for this drugs in different settings or in association with other drugs.

A phase II-III study, actually recruiting, for first line advanced OC, will randomly assigned standard chemotherapy with or without Lurbinectedine (NCT02159820).

Guadecitabine is a DNA demethylating agent, already approved for different tumours. It has been demonstrated that low dose of this drug can resensitize cancer cells to the action of platinum. An Italian phase II study for platinum resistant OC patients have been treated with Decitabine in combination with Carboplatin versus chemotherapy at physician's discretion (NCT01696032), and it showed encouraging activity in heavily pre-treated patients.

More agents are being actually late stage trials, namely NUC-1031, a gemcitabine prodrug, in a phase II trial for platinum-resistant OC (NCT03146663). DEBIO 1143, a second mitochondrial-derived activator of capsases (SMAC mimetic), has been studied in a phase Ib study for OC, and now it is under investigation for the treatment of different solid tumours together with nivolumab, after progression to tanti-PD1 drug (NCT01930292). 
A phase II RCT for patients with resistant/refractory OC, will randomized them to treatment with volasertib-a PLK1 inhibitor, that blocks cell division—or investigator's best choice (NCT01121406).

Adavosertib is a WEE1 tyrosine kinase inhibitor (AZD1775), that acts on G2 phase of cell-cycle. To block this checkpoint means to prevent DNA repair and favour p53-deficient tumour cells not be repaired from DNAdamaging and increase antitumor activity (102). Treatment with adavosertib plus carboplatin showed encouraging activity in patients with $\mathrm{p} 53$-mutated ovarian cancer that is refractory or resistant to first-line chemotherapy (103). Further studies in ovarian cancer are ongoing. One of these, is a phase II trial, for patient with ROC, to test tolerability and efficacy of adavosertib with or without olaparib in treating patients with ROC (NCT03579316).

\section{Conclusions}

Ovarian cancer is a very heterogeneous disease: when OC recurs, it is not a curable disease anymore, with a big variety of clinical, biological, genetic and immunological pathways involved into the response to treatments.

The choice of the most appropriate treatment is based on many factors; if chemotherapy is judged the best option, the choice of single-agent versus combination therapy should take into account many factors: PFI, histotype, molecular characteristics, presence of a BRCA mutation or HRD, number of previous lines of chemotherapy, eventual residual toxicities, nutritional status, symptoms related to the presence of disease $(104,105)$.

Mortality rates remain high, but during the last years antiangiogenics therapies and PARP-inhibitors have changed the history of this disease and improved the outcome in OC patients: as demonstrated by numerous trials and by a recent meta-analysis, PARPi allowed to prolong PFS and OS more than every other previous drug, with wider efficacy in BRCA mutated patients (106). Niraparib have been approved in all patients, regardless of the mutation status, instead Olaparib may be given only to mutated patients as first line and regardless of BRCA status in following recurrences. Rucaparib has been approved both as monotherapy and in a maintenance setting in BRCA mutant tumours.

Among the experimented molecules, ADC binding antigens-as Mirvetuximab soravtansine-are some of the most interesting target agents. These new drugs may contribute to an improved strategy for getting through resistance to standard chemotherapy.

Many combination therapies are actually under trial, similarly as many new target agents; their results will hopefully improve clinical management of this disease and shed some light on many topics: the possibility to rechallenge PARP-i for a second time, after a good response, as in the $\mathrm{OReO}$ trial (NCT03106987), to test the efficacy of PARPis according to HRD status, or to prolong maintenance therapy after oligometastatic relapse, for the hypothetic benefit of continuing treatment beyond progression, to introduce new molecules into clinical practice and better personalize therapy according to patients' need, to reduce side effects and improve QoL. For this purpose questionnaire about QoL should be integrated into routine practice.

\section{Acknowledgments}

Funding: None.

\section{Footnote}

Peer Revieww File: Available at http://dx.doi.org/10.21037/gs20-413

Provenance and Peer Review: This article was commissioned by the Guest Editor (Stefano Cianci) for the series "Ovarian Cancer Recurrence" published in Gland Surgery. The article was sent for external peer review organized by the Guest Editor and the editorial office.

Conflicts of Interest: All authors have completed the ICMJE uniform disclosure form (available at http://dx.doi. org/10.21037/gs-20-413). The series "Ovarian Cancer Recurrence" was commissioned by the editorial office without any funding or sponsorship. The authors have no other conflicts of interest to declare.

Ethical Statement: The authors are accountable for all aspects of the work in ensuring that questions related to the accuracy or integrity of any part of the work are appropriately investigated and resolved.

Open Access Statement: This is an Open Access article distributed in accordance with the Creative Commons Attribution-NonCommercial-NoDerivs 4.0 International License (CC BY-NC-ND 4.0), which permits the noncommercial replication and distribution of the article with 
the strict proviso that no changes or edits are made and the original work is properly cited (including links to both the formal publication through the relevant DOI and the license). See: https://creativecommons.org/licenses/by-nc-nd/4.0/.

\section{References}

1. Torre LA, Trabert B, DeSantis CE, et al. Ovarian Cancer Statistics. CA Cancer J Clin 2018;68:284-96.

2. Jelovac D, Armstrong DK. Recent progress in the diagnosis and treatment of ovarian cancer. CA Cancer J Clin 2011;61:183-203.

3. Parmar MK, Ledermann JA, Colombo N, et al. Paclitaxel plus platinum-based chemotherapy versus conventional platinum-based chemotherapy in women with relapsed ovarian cancer: the ICON4/AGO-OVAR-2.2 trial. Lancet 2003;361:2099-106.

4. The International Collaborative Ovarian Neoplasm (ICON) Group. Paclitaxel plus carboplatin versus standard chemotherapy with either single-agent carboplatin or cyclophosphamide, doxorubicin, and cisplatin in women with ovarian cancer: the ICON3 randomised trial. Lancet 2002;360:505-15.

5. Schmeler KM, Gershenson DM. Low-grade serous ovarian cancer: a unique disease. Curr Oncol Rep 2008;10:519-23.

6. Kyrgiou M, Salanti G, Pavlidis N, et al. Survival benefits with diverse chemotherapy regimens for ovarian cancer: meta-analysis of multiple treatments. J Natl Cancer Inst 2006;98:1655-63.

7. Stuart GC, Kitchener H, Bacon M, et al. 2010 Gynecologic Cancer Intergroup (GCIG) consensus statement on clinical trials in ovarian cancer: report from the fourth Ovarian Cancer Consensus Conference. Int J Gynecol Cancer 2011;21:750-5.

8. Friedlander M, Trimble E, Tinker A, et al. Clinical trials in recurrent ovarian cancer. Int J Gynecol Cancer 2011;21:771-5.

9. Harter P, Hilpertm F, Mahner S, et al. Systemic therapy in recurrent ovarian cancer: current treatment options and new drugs. Expert Rev Anticancer Ther 2010;10:81-8.

10. Eisenhauer EA, Vermorken JB, van Glabbeke M. Predictors of response to subsequent chemotherapy in platinum pretreated ovarian cancer: a multivariate analysis of 704 patients [see comments]. Ann Oncol 1997;8:963-8.

11. Vergote I, Debruyne P, Kridelka F, et al. Phase II study of weekly paclitaxel/carboplatin in combination with prophylactic G-CSF in the treatment of gynecologic cancers: a study in 108 patients by the Belgian
Gynaecological Oncology Group. Gynecol Oncol 2015;138:278-84.

12. Alsop K, Fereday S, Meldrum C, et al. BRCA mutation frequency and patterns of treatment response in BRCA mutation-positive women with ovarian cancer: a report from the Australian ovarian cancer Study Group. J Clin Oncol 2012;30:2654-63.

13. Rafii S, Gourley C, Kumar R, et al. Baseline clinical predictors of antitumor response to the PARP inhibitor olaparib in germline BRCA1/2 mutated patients with advanced ovarian cancer. Oncotarget 2017;8:47154-60.

14. Vergote I, Finkler N, del Campo J, et al. ASSIST-1 study group: phase 3 randomised study of canfosfamide (Telcyta, TLK286) versus pegylated liposomal doxorubicin or topotecan as third-line therapy in patients with platinumrefractory or -resistant ovarian cancer. Eur J Cancer 2009;45:2324-32.

15. Osman MA, Elkady MS, Nasr KE. Weekly paclitaxel versus three-weekly paclitaxel in recurrent platinumresistant epithelial ovarian and peritoneal cancers: a phase III study. Clin Med Insights Oncol 2016;10:35-41.

16. Markman M, Blessing J, Rubin SC, et al. Phase II trial of weekly paclitaxel $(80 \mathrm{mg} / \mathrm{m} 2)$ in platinum and paclitaxelresistant ovarian and primary peritoneal cancers: a Gynecologic Oncology Group study. Gynecol Oncol 2006;101:436-40.

17. Pujade-Lauraine E, Hilpert F, Weber B, et al. Bevacizumab combined with chemotherapy for platinum-resistant recurrent ovarian cancer: the AURELIA open-label randomized phase III trial. J Clin Oncol 2014;32:1302-8.

18. Poveda AM, Selle F, Hilpert F, et al. Bevacizumab combined with weekly paclitaxel, pegylated liposomal doxorubicin, or topotecan in platinum-resistant recurrent ovarian cancer: analysis by chemotherapy cohort of the randomized phase III AURELIA trial. J Clin Oncol 2015;33:3836-8.

19. Pfisterer J, Plante M, Vergote I, et al. Gemcitabine plus carboplatin compared with carboplatin in patients with platinum-sensitive recurrent ovarian cancer: an intergroup trial of the AGO-OVAR, the NCIC CTG, and the EORTC GCG. J Clin Oncol 2006;24:4699-707.

20. Pujade-Lauraine E, Wagner U, Aavall-Lundqvist E, et al. Pegylated liposomal doxorubicin and carboplatin compared with paclitaxel and carboplatin for patients with platinum-sensitive ovarian cancer in late relapse. J Clin Oncol 2010;28:3323-9.

21. Poveda A, Vergote I, Tjulandin S, et al. Trabectedin plus pegylated liposomal doxorubicin in relapsed ovarian 
cancer: outcomes in the partially platinum-sensitive (platinum-free interval 6-12 months) subpopulation of OVA-301 phase III randomized trial. Ann Oncol 2011;22:39-48.

22. Kaye SB, Colombo N, Monk BJ, et al. Trabectedin plus pegylated liposomal doxorubicin in relapsed ovarian cancer delays third-line chemotherapy and prolongs the platinumfree interval. Ann Oncol 2011;22:49-58.

23. Aghajanian C, Blank SV, Goff BA, et al. OCEANS: a randomized, double-blind, placebo-controlled phase III trial of chemotherapy with or without bevacizumab in patients with platinum-sensitive recurrent epithelial ovarian, primary peritoneal, or fallopian tube cancer. J Clin Oncol 2012;30:2039-45.

24. Aghajanian C, Goff B, Nycum LR, et al. Final overall survival and safety analysis of OCEANS, a phase 3 trial of chemotherapy with or without bevacizumab in patients with platinum-sensitive recurrent ovarian cancer. Gynecol Oncol 2015;139:10-6.

25. Shoji T, Eto H, Sato T, et al. A New Therapeutic Strategy for Recurrent Ovarian Cancer-Bevacizumab beyond Progressive Disease. Healthcare (Basel) 2019;7:109.

26. De Lorenzo SB, Patel AG, Hurley RM, et al. The elephant and the blind men: making sense of PARP inhibitors in homologous recombination deficient tumor cells. Front Oncol 2013;3:228.

27. Pennington KP, Walsh T, Harrell MI, et al. Germline and somatic mutations in homologous recombination genes predict platinum response and survival in ovarian, fallopian tube, and peritoneal carcinomas. Clin Cancer Res 2014;20:764-75.

28. Cancer Genome Atlas Research Network. Integrated genomic analyses of ovarian carcinoma. Nature 2011;474:609-15.

29. Scott CL, Swisher EM, Kaufmann SH. Poly (ADPribose) polymerase inhibitors: recent advances and future development. J Clin Oncol 2015;33:1397-406.

30. Fong PC, Boss DS, Yap TA, et al. Inhibition of poly(ADPribose) polymerase in tumors from BRCA mutation carriers. N Engl J Med 2009;361:123-34.

31. Gelmon KA, Tischkowitz M, Mackay H, et al. Olaparib in patients with recurrent high-grade serous or poorly differentiated ovarian carcinoma or triple-negative breast cancer: a phase 2, multicentre, open-label, non-randomised study. Lancet Oncol 2011;12:852-61.

32. Ledermann J, Harter P, Gourley C, et al. Olaparib maintenance therapy in patients with platinum-sensitive relapsed serous ovarian cancer: a preplanned retrospective analysis of outcomes by BRCA status in a randomised phase 2 trial. Lancet Oncol 2014;15:852-61.

33. Coleman RL, Oza AM, Lorusso D, et al. Rucaparib maintenance treatment for recurrent ovarian carcinoma after response to platinum therapy (ARIEL3): a randomised, double-blind, placebo-controlled, phase 3 trial. Lancet 2017;390:1949-61.

34. Patel AG, Sarkaria JN, Kaufmann SH. Nonhomologous end joining drives poly (ADP-ribose) polymerase (PARP) inhibitor lethality in homologous recombination-deficient cells. Proc Natl Acad Sci USA 2011;108:3406-11.

35. Murai J, Huang SY, Das BB, et al. Trapping of PARP1 and PARP2 by clinical PARP inhibitors. Cancer Res 2012;72:5588-99.

36. Pujade-Lauraine E, Ledermann JA, Selle F, et al. Olaparib tablets as maintenance therapy in patients with platinumsensitive, relapsed ovarian cancer and BRCA 1/2 mutation (SOLO2/ENGOT-OV21): a double randomised, placebocontrolled, phase 3 trial. Lancet Oncol 2017;18:1274-84.

37. Friedlander M, Gebski V, Gibbs E, et al. Health related quality of life and patient-centred outcomes with olaparib maintenance after chemotherapy in patients with platinumsensitive, relapsed ovarian cancer and a BRCA1/2 mutation (SOLO2/ENGOT Ov-21): a placebo-controlled, phase 3 randomised trial. Lancet Oncol 2018;19:1126-34.

38. Moore K, Colombo N, Scambia G, et al. Maintenance Olaparib in Patients with Newly Diagnosed Advanced Ovarian Cancer. N Engl J Med 2018;379:2495-505.

39. FDA, Lynparza (olaparib) tablets, for oral use, Highlights of Prescribing Information, 2014.

40. Evans T, Matulonis U. PARP inhibitors in ovarian cancer: evidence, experience and clinical potential. Ther Adv Med Oncol 2017;9:253-67.

41. Ledermann J, Harter P, Gourley C, et al. Olaparib maintenance therapy in platinum-sensitive relapsed ovarian cancer. N Engl J Med 2012;366:1382-92.

42. Mirza MR, Monk BJ, Herrstedt J, et al. Niraparib maintenance therapy in platinum-sensitive, recurrent ovarian cancer. N Engl J Med 2016;375:2154-64.

43. Moore KN, Secord AA, Geller MA, et al. Niraparib monotherapy for late-line treatment of ovarian cancer (QUADRA): a multicentre, open-label, single-arm, phase 2 trial. Lancet Oncol 2019;20:636-48.

44. Oza AM, Matulonis UA, Malander S et al. Quality of life in patients with recurrent ovarian cancer treated with niraparib versus placebo (ENGOT-OV16/NOVA): results from a double-blind, phase 3 , randomised controlled trial. Lancet Oncol 2018;19:1117-25. 
45. FDA, Zejula (niraparib) capsules, for oral use. Highlights of prescribing information, 2017.

46. Berek JS, Matulonis UA, Peen U, et al. Safety and dose modification for patients receiving niraparib. Ann Oncol 2018;29:1784-92.

47. FDA, Rubraca (rucaparib) tablets, for oral use. Highlights of Prescribing Information, 2016.

48. Swisher EM, Lin KK, Oza AM, et al. Rucaparib in relapsed, platinumsensitive high-grade ovarian carcinoma (ARIEL2 part 1): an international, multicentre, open-label, phase 2 trial. Lancet Oncol 2017;18:75-87.

49. Kristeleit RS, Oaknin A, Ray-Coquard I, et al. Antitumor activity of the poly(ADP-ribose) polymerase inhibitor rucaparib as monotherapy in patients with platinumsensitive, relapsed, BRCA-mutated, high-grade ovarian cancer, and an update on safety. Int J Gynecol Cancer 2019;29:1396-404.

50. Konecny GE, Oza AM, Tinker AV, et al. Rucaparib in patients with relapsed, primary platinum-sensitive highgrade ovarian carcinoma with germline or somatic BRCA mutations: Integrated summary of efficacy and safety from the phase II study ARIEL2. Gynecol Oncol 2017;145:2 .

51. Reiss KA, Herman JM, Armstrong D, et al. A final report of a phase I study of veliparib (ABT-888) in combination with low-dose fractionated whole abdominal radiation therapy (LDFWAR) in patients with advanced solid malignancies and peritoneal carcinomatosis with a dose escalation in ovarian and fallopian tube cancers. Gynecol Oncol 2017;144:486-90.

52. de Bono J, Ramanathan RK, Mina L, et al. Phase I, dose-escalation, two-part trial of the PARP inhibitor talazoparib in patients with advanced germline BRCA1/2 mutations and selected sporadic cancers. Cancer Discov 2017;7:620-9.

53. Coleman RL, Sill MW, Bell-McGuinn K, et al. A phase II evaluation of the potent, highly selective PARP inhibitor veliparib in the treatment of persistent or recurrent epithelial ovarian, fallopian tube, or primary peritoneal cancer in patients who carry a germline BRCA1 or BRCA2 mutation - an NRG oncology/Gynecologic Oncology Group study. Gynecol Oncol 2015;137:386-91.

54. Coleman RL, Fleming GF, Brady MF, et al. Veliparib with First-Line Chemotherapy and as Maintenance Therapy in Ovarian Cancer. N Engl J Med 2019;381:2403-15.

55. Strickland KC, Howitt BE, Shukla SA, et al. Association and prognostic significance of BRCA1 of BRCA1/2mutation status with neoantigen load, number of tumorinfiltrating lymphocytes and expression of PD-1/PD-
L1 in high grade serous ovarian cancer. Oncotarget 2016;7:13587-98.

56. Hamanishi J, Mandai M, Ikeda T, et al. Safety and antitumor activity of anti-PD-1 antibody, nivolumab, in patients with platinum-resistant ovarian cancer. J Clin Oncol 2015;33:4015-22.

57. Varga A, Piha-Paul S, Ott PA, et al. Pembrolizumab in patients with programmed death ligand 1-positive advanced ovarian cancer: Analysis of KEYNOTE-028. Gynecol Oncol 2019;152:243-50.

58. Disis ML, Patel MR, Pant S, et al. Avelumab (MSB0010718C), an anti-PD-L1 antibody, in patients with previously treated, recurrent or refractory ovarian cancer: a phase Ib, open-label expansion trial. J Clin Oncol 2015;33:5509.

59. Infante JR, Braiteh F, Emens LA, et al. Safety, clinical activity and biomarkers of atezolizumab (atezo) in advanced ovarian cancer (OC). Ann Oncol 2016;27:296-312.

60. Sun LM, Liu YC, Li W, et al. Nivolumab effectively inhibit platinum-resistant ovarian cancer cells via induction of cell apoptosis and inhibition of ADAM17 expression. Eur Rev Med Pharmacol Sci 2017;21:1198-205.

61. Schmitt J, Matei D. Targeting angiogenesis in ovarian cancer. Cancer Treat Rev 2012;38:272-83.

62. Ledermann JA, Embleton AC, Raja F, et al. Cediranib in patients with relapsed platinum-sensitive ovarian cancer (ICON6): a randomised, double-blind, placebo-controlled phase 3 trial. Lancet 2016;387:1066-74.

63. Ledermann JA, Embleton AC, Perren T, et al. Overall survival results of ICON6: A trial of chemotherapy and cediranib in relapsed ovarian cancer. J Clin Oncol 2017;35:5506.

64. Stark DP, Cook A, Brown JM, et al. Quality of life with cediranib in relapsed ovarian cancer: the ICON6 phase 3 randomized clinical trial. Cancer 2017;123:2752-61.

65. Huang J, Bae JO, Tsai JP, et al. Angiopoietin-1/Tie-2 activation contributes to vascular survival and tumor growth during VEGF blockade. Int J Oncol 2009;34:79-87.

66. Monk BJ, Poveda A, Vergote I, et al. Final results of a phase 3 study of trebananib plus weekly paclitaxel in recurrent ovarian cancer (TRINOVA-1): long-term survival, impact of ascites, and progression-free survival-2. Gynecol Oncol 2016;143:27-34.

67. Monk BJ, Poveda A, Vergote I, et al. Anti-angiopoietin therapy with trebananib for recurrent ovarian cancer (TRINOVA-1): a randomised, multicentre, doubleblind, placebo-controlled phase 3 trial. Lancet Oncol 2014;15:799-808. 
68. Marth C, Vergote I, Scambia G, et al. ENGOT-ov-6/ TRINOVA-2: randomised, double-blind, phase 3 study of pegylated liposomal doxorubicin plus trebananib or placebo in women with recurrent partially platinumsensitive or resistant ovarian cancer. Eur J Cancer 2017;70:111-21.

69. Friedlander M, Hancock KC, Rischin D, et al. A phase II, open-label study evaluating pazopanib in patients with recurrent ovarian cancer. Gynecol Oncol 2010;119:32-7.

70. Pignata S, Lorusso D, Scambia G, et al. MITO 11 investigators. Pazopanib plus weekly paclitaxel versus weekly paclitaxel alone for platinum-resistant or platinumrefractory advanced ovarian cancer (MITO 11): a randomised, open-label, phase 2 trial. Lancet Oncol 2015;16:561-8.

71. Vergote I, du Bois A, Floquet A, et al. Overall survival results of AGO-OVAR16: A phase 3 study of maintenance pazopanib versus placebo in women who have not progressed after first-line chemotherapy for advanced ovarian cancer. Gynecol Oncol 2019;155:186-91.

72. Kummar S, Oza AM, Fleming GF, et al. Randomized trial of Oral cyclophosphamide and Veliparib in highgrade serous ovarian, primary peritoneal, or fallopian tube cancers, or BRCA-mutant ovarian Cancer. Clin Cancer Res 2015;21:1574-82.

73. Bell-McGuinn KM, Brady WE, Schilder RJ, et Al. A phase I study of continuous veliparib in combination with IV carboplatin/paclitaxel or IV/IP paclitaxel/cisplatin and bevacizumab in newly diagnosed patients with previously untreated epithelial ovarian, fallopian tube, or primary peritoneal cancer: An NRG Oncology/Gynecologic Oncology Group study. J Clin Oncol 2015;33:5507.

74. Lee JM, Hays JL, Annunziata CM, et al. Phase I/Ib study of olaparib and carboplatin in BRCA1 or BRCA2 mutation associated breast or ovarian cancer with biomarker analyses. J Natl Cancer Inst 2014;106:dju089.

75. Kummar S, Chen A, Ji J, et al. Phase I study of PARP inhibitor ABT-888 in combination with topotecan in adults with refractory solid tumors and lymphomas. Cancer Res 2011;71:5626-34.

76. LoRusso PM, Li J, Burger A, et al. Phase I Safety, Pharmacokinetic, and Pharmacodynamic Study of the Poly(ADP-ribose) Polymerase (PARP) Inhibitor Veliparib (ABT-888) in Combination with Irinotecan in Patients with Advanced Solid Tumors. Clin Cancer Res 2016;22:3227-37.

77. Tan A, Toppmeyer D, Stein MN, et al. Phase I trial of veliparib (ABT-888): a poly (ADP-ribose) polymerase
(PARP) inhibitor in combination with doxorubicin and cyclophosphamide in breast cancer and other solid tumors. J Clin Oncol 2011;29:3041.

78. Gray HJ, Bell-McGuinn K, Fleming GF, et al. Phase I combination study of the PARP inhibitor veliparib plus carboplatin and gemcitabine in patients with advanced ovarian cancer and other solid malignancies. Gynecol Oncol 2018;148:507-14.

79. Wilson RH, Evans TJ, Middleton MR, et al. A phase I study of intravenous and oral rucaparib in combination with chemotherapy in patients with advanced solid tumours. Br J Cancer 2017;116:884-92.

80. Cortez AJ, Tudrej P, Kujawa KA, et al. Advances in ovarian cancer therapy. Cancer Chemother Pharmacol 2018;81:17-38.

81. Jiao S, Xia W, Yamaguchi H, et al. PARP inhibitor upregulates PD-L1 expression and enhances cancerassociated immunosuppression. Clin Cancer Res 2017;23:3711-20.

82. Hegan DC, Lu Y, Stachelek GC, et al. Inhibition of poly(ADP-ribose) polymerase down-regulates BRCA1 and RAD51 in a pathway mediated by E2F4 and p130. Proc Natl Acad Sci USA 2010;107:2201-6.

83. Liu JF, Barry WT, Birrer M, et al. Combination cediranib and olaparib versus olaparib alone for women with recurrent platinum-sensitive ovarian cancer: a randomised phase 2 study. Lancet Oncol 2014;15:1207-14.

84. Mirza MR, Bergmann TK, Mau-Sørensen M, et al. A phase I study of the PARP inhibitor niraparib in combination with bevacizumab in platinum-sensitive epithelial ovarian cancer: NSGO AVANOVA1/ENGOTOV24. Cancer Chemother Pharmacol 2019;84:791-8.

85. Colombo N, Nicoletto MO, Benedetti Panici P, et al. Barocco: a randomized phase II study of weekly paclitaxel versus cediranib-olaparib combination given with continuous or intermittent schedule in patients with recurrent platinum resistant ovarian cancer (PROC). Ann Oncol 2019;30:851-934.

86. Ray-Coquard I, Selle F, Harter P, et al. PAOLA-1: An ENGOT/GCIG phase III trial of olaparib versus placebo combined with bevacizumab as maintenance treatment in patients with advanced ovarian cancer following first-line platinum-based chemotherapy plus bevacizumab. J Clin Oncol 2016;34:TPS5607.

87. Petrillo M, De Iaco P, Cianci S, et al. Long-Term Survival for Platinum-Sensitive Recurrent Ovarian Cancer Patients Treated with Secondary Cytoreductive Surgery Plus Hyperthermic Intraperitoneal Chemotherapy (HIPEC). 
Ann Surg Oncol 2016;23:1660-5.

88. Cianci S, Abatini C, Fagotti A, et al. Hyperthermic intraperitoneal chemotherapy (HIPEC) for peritoneal malignancies using new hybrid CO2 system: preliminary experience in referral center. Updates Surg 2019;71:555-60.

89. Fagotti A, Costantini B, Gallotta V, et al. Minimally invasive secondary cytoreduction plus HIPEC versus open surgery plus HIPEC in isolated relapse from ovarian cancer: a retrospective cohort study on perioperative outcomes. J Minim Invasive Gynecol 2015;22:428-32.

90. Sugarbaker PH. Surgical management of peritoneal carcinosis: diagnosis, prevention and treatment. Langenbecks Arch Chir 1988;373:189-96.

91. Spiliotis J, Vaxevanidou A, Sergouniotis F, et al. The role of cytoreductive surgery and hyperthermic intraperitoneal chemotherapy in the management of recurrent advanced ovarian cancer: a prospective study. J BUON 2011;16:74-9.

92. Harter P, Reuss A, Sehouli J, et al. Brief report about the role of hyperthermic intraperitoneal chemotherapy in a prospective randomized phase 3 Study in recurrent ovarian cancer from Spiliotis et al. Int J Gynecol Cancer 2017;27:246-7.

93. Batista TP. Comment on: surgery and HIPEC in recurrent epithelial ovarian cancer: a prospective randomized phase III study. Ann Surg Oncol 2017;24:630.

94. Chiva LM, Gonzalez-Martin A. A critical appraisal of hyperthermic intraperitoneal chemotherapy (HIPEC) in the treatment of advanced and recurrent ovarian cancer. Gynecol Oncol 2015;136:130-5.

95. Lim MC, Chang SJ, Yoo HJ, et al. Randomized trial of hyperthermic intraperitoneal chemotherapy (HIPEC) in women with primary advanced peritoneal, ovarian, and tubal cancer. J Clin Oncol 2017;35:5520.

96. Hotouras A, Desai D, Bhan C, et al. Heated intraperitoneal chemotherapy (HIPEC) for patients with recurrent ovarian cancer: a systematic literature review. Int J Gynecol Cancer 2016;26:661-70.

97. Harter P, du Bois A, Mahner S, et al. Statement of the AGO Kommission Ovar, AGO Study Group, NOGGO, AGO Austria and AGO Switzerland Regarding the Use of Hyperthermic Intraperitoneal Chemotherapy (HIPEC) in Ovarian Cancer. Geburtshilfe Frauenheilkd 2016;76:147-9.

98. Colombo N, Sessa C, Bois AD, et al. ESMO-ESGO consensus conference recommendations on ovarian cancer: pathology and molecular biology, early and advanced stages, borderline tumours and recurrent disease. Int J Gynecol Cancer 2019. doi: 10.1136/ijgc-2019-000308.

99. Moore KN, Martin LP, O'Malley DM, et al. Safety and activity of mirvetuximab soravtansine (IMGN853), a folate receptor alpha-targeting antibody-drug conjugate, in platinum-resistant ovarian, fallopian tube, or primary peritoneal cancer: a phase I expansion study. J Clin Oncol 2017;35:1112-8.

100.Poveda A, Del Campo JM, Ray-Coquard I, et al. II randomized study of PM01183 versus topotecan in patients with platinum-resistant/refractory advanced ovarian cancer. Ann Oncol 2017;28:1280-7.

101. Gaillard S, Ghamande SA, Pardo B, et al. CORAIL trial: randomized phase III study of lurbinectedin (PM01183) versus pegylated liposomal doxorubicin (PLD) or topotecan (T) in patients with platinum-resistant ovarian cancer. J Clin Oncol 2016;34:TPS5597.

102. Mahajan K, Mahajan NP. WEE1 tyrosine kinase, a novel epigenetic modifier. Trends Genet 2013;29:394-402.

103. Leijen S, Van Geel R, Sonke GS, et al. Phase II study with wee1 inhibitor AZD1775 (MK-1775) plus carboplatin in patients with p53 mutated ovarian cancer refractory or resistant ( $<3$ months) to standard first line therapy. J Clin Oncol 2015;33:2507.

104.Corrado G, Salutari V, Palluzzi E, et al. Optimizing treatment in recurrent epithelial ovarian cancer. Expert Rev Anticancer Ther 2017;17:1147-58.

105. Cianci S, Rumolo V, Rosati A, et al. Sarcopenia in Ovarian Cancer Patients, Oncologic Outcomes Revealing the Importance of Clinical Nutrition: Review of Literature. Curr Pharm Des 2019;25:2480-90.

106. Tomao F, Bardhi E, Di Pinto A, et al. Parp inhibitors as maintenance treatment in platinum sensitive recurrent ovarian cancer: An updated meta-analysis of randomized clinical trials according to BRCA mutational status. Cancer Treatment Reviews 2019;80:101909.

Cite this article as: Mancari R, Cutillo G, Bruno V, Vincenzoni C, Mancini E, Baiocco E, Bruni S, Vocaturo G, Chiofalo B, Vizza E. Development of new medical treatment for epithelial ovarian cancer recurrence. Gland Surg 2020;9(4):1149-1163. doi $10.21037 / g s-20-413$ 